\title{
Expression and clinical significance of thrombospondin-1 and plasminogen activator inhibitor-1 in patients with mesangial proliferative glomerulonephritis
}

\author{
Cuiping Pan, Canxin Zhou, Aiqin Shan, Na Chen \\ Department Nephrology, Ningbo Yinzhou People's Hospital, Ningbo, China \\ Contributions: (I) Conception and design: C Pan; (II) Administrative support: C Zhou; (III) Provision of study materials or patients: C Pan, A Shan, N \\ Chen; (IV) Collection and assembly of data: All authors; (V) Data analysis and interpretation: C Pan, C Zhou, A Shan; (VI) Manuscript writing: All \\ authors; (VII) Final approval of manuscript: All authors. \\ Correspondence to: Cuiping Pan. Baizhang East Road No. 251, Yinzhou District, Ningbo, China. Email: pancuiping0205@163.com.
}

Background: As a common pathological type of glomerular disease in China, mesangial proliferative glomerulonephritis is related to plasminogen activator inhibitor-1 (PAI-1) and thrombospondin-1 (TSP-1). Here, this study aims to investigate the expression and clinical significance of TSP-1 and PAI-1 in patients with mesangial proliferative glomerulonephritis.

Methods: Renal tissue specimens from 46 patients with mesangial proliferative glomerulonephritis admitted to this hospital were selected as the subjects, and 8 specimens of renal tissue from autopsy were used as controls. The expression levels of TSP-1 and PAI-1 were detected by immunohistochemistry and analyzed.

Results: The 24-hour urine protein, triglyceride, and total cholesterol levels of patients with severe mesangial hyperplasia were significantly higher than those of patients with mild and moderate mesangial hyperplasia, and serum albumin was lower than that of patients with mild and moderate mesangial hyperplasia $(\mathrm{P}<0.05)$. The 24-hour urine protein level of patients with moderate mesangial hyperplasia was higher than that of patients with mild mesangial hyperplasia while the albumin level was lower $(\mathrm{P}<0.05)$, but there was no significant difference in triglyceride and total cholesterol $(\mathrm{P}>0.05)$. There was no significant difference in creatinine clearance (Ccr) between the three groups $(\mathrm{P}>0.05)$. The 24-hour urine protein and urine alpha-1-microglobulin (A1M) levels in patients with renal interstitial disease were higher than those in patients without renal interstitial disease, while their Ccr level was lower $(\mathrm{P}<0.05)$. TSP-1 and PAI-1 were not positively expressed in the glomeruli and renal tubules of specimens of the control group. However, in mesangial hyperplasia patients, the expression of TSP-1 and PAI-1 in mesangial hyperplasia with varying degrees and in different renal tubular damage were as follows: mild degree $<$ moderate degree $<$ severe degree $(\mathrm{P}<0.05)$.

Conclusions: The pathological changes of mesangial proliferative glomerulonephritis are related to 24-hour urine protein, triglyceride, total cholesterol level, urine A1M, and Ccr level. The expression of TSP-1 and PAI-1 in the mesenchyme of glomerular and renal tubules significantly increased with the severity of the disease, suggesting that TSP-1 and PAI-1 play an important role in the occurrence and development of mesangial proliferative glomerulonephritis.

Keywords: Thrombospondin-1 (TSP-1); plasminogen activator inhibitor-1 (PAI-1); mesangial proliferative glomerulonephritis; immunohistochemistry

Submitted May 21, 2020. Accepted for publication Aug 18, 2020.

doi: 10.21037/apm-20-1291

View this article at: http://dx.doi.org/10.21037/apm-20-1291 


\section{Introduction}

Mesangial proliferative glomerulonephritis is a common pathological type of glomerular disease in China (1), characterized by diffuse mesangial cell proliferation and/ or increased mesangial matrix. The etiology of this disease is not clear yet, but some studies have reported that it is a severe immune-mediated inflammatory disease, caused by immune circulation complex deposition in the glomerular mesangial through some antigens to stimulate the body to produce antibodies (2). As a member of serine protease inhibitors family, plasminogen activator inhibitor-1 (PAI1 ) is a kind of inflammatory proteins produced by various cells, which can restrain fibrin degradation and promote the deposition of fibrin. PAI-1 can also affect the synthesis and degradation balance of extracellular matrix (ECM) of the glomerulus and promote glomerulosclerosis. Thrombospondin-1 (TSP-1), which is distributed in renal tissues, renal tubules and mesangial cells, is one of the most important extracellular matrix glycoproteins that can lead to fibrosis $(3,4)$. In recent years, a lot of studies have shown that both of the molecules have certain effect on the occurrence of mesangial proliferative glomerulonephritis $(5,6)$. Therefore, to further investigate the clinical significance of TSP-1 and PAI-1 expression in patients with mesangial proliferative glomerulonephritis, the renal tissue specimens from 46 patients with mesangial proliferative glomerulonephritis who were admitted to our nephrology department from May 2013 to May 2018 were collected and studied. We present the following article in accordance with the MDAR reporting checklist (available at http://dx.doi. org/10.21037/apm-20-1291).

\section{Methods}

\section{General information}

The 46 subject patients with mesangial proliferative glomerulonephritis were selected from our nephrology department from May 2013 to May 2018. Specimens were obtained using renal puncture biopsies, and were then fixed and paraffin-embedded. All patients were subjected to renal biopsy histopathology to confirm the diagnosis. There were 28 males and 18 females, aged 19 to 56 years old, with an average age of $34.75 \pm 6.24$ years old and a disease course of 1 month to 9 years, with an average of $14.5 \pm 6.31$ months. Of the 49 patients, 16 showed nephrotic syndrome, 25 showed chronic glomerulonephritis, and 5 showed occult nephritis. There were 8 cases with hematuria, 20 cases with elevated blood pressure, 37 cases with edema, 6 cases with nausea, and 16 cases with soreness of the loins. There were 16 cases without cause before the onset, 26 cases with upper respiratory tract infection, and 4 cases with urinary tract infection.

For the control group, 8 autopsy kidney tissue specimens from patients who died unexpectedly between May 2013 and May 2018 were selected. The patients had no renal tissue lesions or damage, showed normal renal function, and met medical ethics requirements. There were 5 males and 3 females, aged 23 to 59 years old, with an average age of $36.00 \pm 5.96$ years old.

Inclusion criteria: (I) complete clinical data; (II) aged between 19 and 59 years old; (III) no diabetes history. Exclusion criteria: (I) impaired renal function and abnormal thyroid metabolism; (II) patients had diabetic ketoacidosis recently; (III) patients who had taken thyroid hormone metabolism or kidney function drugs.

The study was approved by Ningbo Yinzhou People's Hospital Community (No. 2019-046). All procedures performed in this study involving human participants were in accordance with the Declaration of Helsinki (as revised in 2013). The informed consent was taken from all the patients.

\section{Research methods}

\section{Main reagents}

Primary antibodies for rabbit anti-TSP-1 antibody, mouse anti-human PAI-1 antibody, and immunohistochemical staining kit (ready-to-use) were purchased from Baoshide Company (Wuhan, China).

\section{Methodology}

TSP-1 and PAI-1 immunohistochemical staining was performed by strept avidin biotin-peroxidase complex (SABC) staining method (7). Tissue sections were routinely dewaxed and rinsed, and 3\% methanol hydrogen peroxide was added for 20 minutes. Samples from the same patients were divided into two groups: one group was subjected to TSP-1 immunohistochemical staining, and the other group was subjected to PAI-1 immunohistochemical staining. The operations were conducted strictly according to the kit instructions. The results of TSP-1 and PAI-1 staining of known mesangial proliferative glomerulonephritis tissues were used as the positive control, while the product of replacing the primary and secondary antibodies with phosphate-buffered saline (PBS) solution was used the 
negative control.

\section{Methods for detecting renal function indicators}

Four $\mathrm{mL}$ of fasting venous blood were collected from all the patients in the 3 groups at $8 \mathrm{am}$ in the morning to detect serum albumin, triglycerides, and total cholesterol levels. The enzymatic method was used to determine serum Scr level. The 24-hour urine output samples of patients in the 3 groups of need to be collected excluding proteinuria caused by exercise, infection and other factors, and the 24-hour proteinuria was measured with toluene preservation methods. Urine a-1 microglobulin in the urine 1 hour after drinking $5,000 \mathrm{~mL}$ of water at 6 am was detected by radioimmunoassay (RIA).

Ccr calculation formula: [(140-age $) \times$ body weight $(\mathrm{kg})] /$ $[0.818 \times$ serum creatinine $(\mu \mathrm{g} / \mathrm{mL})]$.

\section{Criteria of pathological scoring}

Following the 1982 classification criteria for glomerular pathology from the World Health Organization (WHO) (8), the scoring assessment of glomerular pathology in our study considered three criteria: mesangial cell proliferation, stroma widening, and changes in sclerosis. These were recorded from 0 to 3 points according to normal, mild, moderate, and severe levels, respectively. A mild level was defined as slight proliferation of mesangial cells, no significant effect on capillary ridges, and glomerular sclerosis $<30 \%$. A moderate level was defined as moderate proliferation of mesangial cells, occlusion of capillary narrowing $<50 \%$, and glomerular sclerosis of $30-60 \%$. A severe level was defined as severe proliferation of mesangial cells mesangial cells, occlusion of capillary narrowing $>50 \%$, and glomerular sclerosis $>60 \%$.

The scoring for the mesenchyme of renal tubule pathology included four criteria: renal tubular degeneration, atrophy, interstitial inflammatory cell infiltration, and interstitial fibrosis. A mild level was defined as slightly damaged renal tubules with small focal tubular atrophy, interstitial inflammatory cell infiltration, and interstitial fibrosis. A moderate level was defined as moderately damaged renal tubules and focal tubular atrophy, interstitial inflammatory cell infiltration, and interstitial fibrosis. A severe level was defined as severely damaged renal tubules and diffuse tubular atrophy, interstitial inflammatory cell infiltration, and interstitial fibrosis. These were recorded from 0 to 3 points according to normal, mild, moderate and severe degree, respectively.
Scores from glomerular pathology assessment were totaled, and specimens were defined as mild ( $0-4$ points), moderate (5-8 points), and severe (9-12 points). Scores for renal interstitial pathological damage were also totaled, and specimens were defined as mild ( $0-4$ points), moderate (5-8 points), and severe (9-12 points).

\section{Statistical methods}

All data were performed using SPSS 18.0 software. Measurement data are described as mean \pm standard deviation $(\bar{x} \pm S)$, and were analyzed by $t$-test or variance test. Count data and expressed as rate/composition ratio, and the comparison between groups was assessed by $\chi^{2}$ test. Results with a $\mathrm{P}$ value $<0.05$ were considered statistically significant.

\section{Results}

\section{Relationship between mesangial hyperplasia and clinical indicators}

The 24-hour urine protein, triglyceride, and total cholesterol levels of patients with severe mesangial hyperplasia were significantly higher than those of patients with mild and moderate mesangial hyperplasia, while the serum albumin was lower than that of patients with mild and moderate mesangial hyperplasia $(\mathrm{P}<0.05)$. The 24-hour urine protein level of patients with moderate mesangial hyperplasia was higher than that of patients with mild mesangial hyperplasia, while the albumin level was lower than that $(\mathrm{P}<0.05)$, but there was no significant difference in triglyceride and total cholesterol $(\mathrm{P}>0.05)$. There was also no significant difference in creatinine clearance (Ccr) between the three groups $(\mathrm{P}>0.05)$ (Table 1).

\section{Relationship between renal interstitial lesion and renal function}

The 24-hour urinary protein and urine A1M levels of patients with renal interstitial lesions were higher than those without renal interstitial lesions, while Ccr levels were lower $(\mathrm{P}<0.05)($ Table 2).

\section{Comparison of TSP-1 expression level in mesangial proliferative glomerulonephritis and normal renal tissue specimens}

TSP-1 was not positively expressed in the glomeruli and 


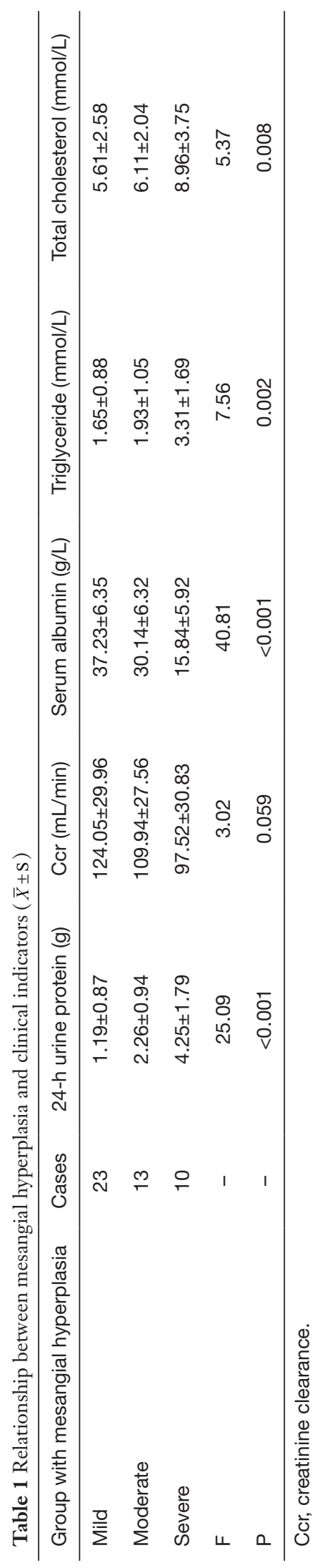

renal tubules of the control group, while its expression in mesangial hyperplasia patients increased with the severity of renal tubular damage $(\mathrm{P}<0.05)$ (Table 3).

\section{Comparison of PAI-1 expression levels between the mesangial proliferative glomerulonephritis and normal renal tissue specimens}

There was no positive expression of PAI-1 in the glomeruli and tubules of the control group, while its expression in mesangial hyperplasia patients increased with the severity of tubular damage $(\mathrm{P}<0.05)$ (Table 4).

\section{Discussion}

Mesangial proliferative glomerulonephritis is a pathological subtype of glomerulonephritis and is common in China and Australia, particularly in adolescents (9). Although most patients have a good prognosis, renal function decline occurs in some patients and may even progress to endstage renal failure (10). This disease is mostly induced by upper respiratory tract infections with diverse clinical manifestations, most of which are primary nephrotic syndrome (11), asymptomatic hematuria, proteinuria, and other non-specific symptoms, so clinical diagnosis and prognosis are problematic. At present, the etiology, pathology, and pathogenesis of the disease are not clear and there are few studies on the expression of PAI- 1 and TSP1 in mesangial proliferative glomerulonephritis. Thus, in order to guide clinical diagnosis and treatment, clinicians rely on finding sensitive and specific protein molecules as biomarkers of pathological changes. Most studies have shown that glomerular capillary endothelial injury and coagulopathy are important factors in the occurrence of this disease (12-14), which causes diffuse thrombosis in the glomerulus where ECM deposition arising from an imbalance of the plasmin system is a key precipitator of glomerulosclerosis (15-17).

The results of this study showed that the 24-hour urinary protein, triglycerides, and total cholesterol levels in patients with severe mesangial hyperplasia were significantly higher than those in patients with mild and moderate mesangial hyperplasia, while the serum albumin level was lower. The 24-hour urine protein in patients with moderate mesangial hyperplasia was higher than that in patients with mild mesangial hyperplasia, while the albumin levels were lower, but there was no significant difference in triglyceride and total cholesterol levels. Furthermore, there was no 
Table 2 Relationship between renal interstitial lesions and renal function $(\bar{X} \pm \mathrm{S})$

\begin{tabular}{lcccc}
\hline Group & Cases & 24-h urinary protein quantification $(\mathrm{g})$ & Urine A1M $(\mathrm{mg} / \mathrm{L})$ & $\mathrm{Ccr}(\mathrm{mL} / \mathrm{min})$ \\
\hline Renal interstitial lesion & 25 & $1.87 \pm 1.07$ & $286.79 \pm 154.31$ & $125.35 \pm 28.66$ \\
No renal interstitial lesion & 21 & $3.56 \pm 1.58$ & $576.61 \pm 204.77$ & $89.74 \pm 37.98$ \\
$\mathrm{t}$ & - & 4.305 & 5.469 & 3.621 \\
$\mathrm{P}$ & - & $<0.001$ & $<0.001$ & 0.001 \\
\hline
\end{tabular}

Ccr, creatinine clearance; A1M, alpha-1-microglobulin.

Table 3 Comparison of TSP-1 level in mesangial proliferative glomerulonephritis and normal renal tissue specimens $(\bar{X} \pm \mathrm{S})$

\begin{tabular}{|c|c|c|c|c|}
\hline Variable & \multicolumn{2}{|c|}{ Group with mesangial hyperplasia } & \multicolumn{2}{|c|}{ Group with renal interstitial lesion } \\
\hline Control & 8 & 0 & 8 & 0 \\
\hline Mile & 23 & $0.38 \pm 0.08$ & 10 & $0.76 \pm 0.18$ \\
\hline Moderate & 13 & $0.49 \pm 0.10$ & 5 & $1.05 \pm 0.19$ \\
\hline F (last three groups) & - & 121.48 & - & 83.03 \\
\hline P (last three groups) & - & $<0.001$ & - & $<0.001$ \\
\hline
\end{tabular}

TSP-1, thrombospondin-1.

Table 4 Comparison of PAI-1 levels between mesangial proliferative glomerulonephritis and normal renal tissue specimens $(\bar{X} \pm \mathrm{S})$

\begin{tabular}{lcccc}
\hline \multirow{2}{*}{ Variable } & \multicolumn{2}{c}{ Group with mesangial hyperplasia } & \multicolumn{2}{c}{ Group with renal interstitial lesion } \\
\cline { 2 - 5 } & Cases & PAl-1 level $(\mu \mathrm{g} / \mathrm{mL})$ & Cases & \multicolumn{2}{c}{$\mathrm{PAl}-1$ level $(\mu \mathrm{g} / \mathrm{mL})$} \\
\hline Control & 8 & 0 & 10 & 0 \\
Mile & 23 & $0.15 \pm 0.10$ & 5 & $0.70 \pm 0.18$ \\
Moderate & 13 & $0.23 \pm 0.12$ & 6 & $1.42 \pm 0.24$ \\
Severe & 10 & $0.41 \pm 0.09$ & - & 23.70 \\
F (last three groups) & - & 21.75 & - & $<0.001$ \\
P (last three groups) & - & $<0.001$ &
\end{tabular}

PAl-1, plasminogen activator inhibitor-1.

significant difference in Ccr between the three groups. The expression levels of urinary protein quantification and urine $\mathrm{A} 1 \mathrm{M}$ in patients with renal interstitial disease were higher than those without renal interstitial disease, while the Ccr level was lower. This indicates that the more severe the glomerular pathological changes in patients is, the greater the difference between clinical indicators like 24-hour urine protein quantification, triglycerides, total cholesterol, and serum albumin from the normal range will be. Patients with renal interstitial disease may even suffer impaired renal function, which suggests that the degree of pathological changes could reflect the degree of the patient's condition. Therefore, it is of great importance to assess the degree of pathological changes of the glomeruli and renal interstitium in clinic $(18,19)$.

Previous studies have shown that highly expressed PAI1 and TSP- 1 can be found in the mesangial region and renal tubulointerstitium of this disease, suggesting that 
they are closely related to the pathogenesis of mesangial proliferative glomerulonephritis (20). In this study, TSP1 and PAI-1 were not positively expressed in normal renal tissue specimens, and the expression level of them in different degrees of mesangial hyperplasia and different degrees of renal tubular injury was mild $<$ moderate $<$ severe. Therefore, we can perhaps conclude that TSP-1 and PAI-1 are inextricably linked to pathological changes of mesangial hyperplasia and tubulointerstitial lesions; they may promote the progression of mesangial proliferative glomerulonephritis, and their expression levels could thus indirectly reflect the disease progression.

In summary, the pathological changes of mesangial proliferative glomerulonephritis are related to clinical indicators of 24-hour urine protein quantification, triglycerides, total cholesterol, urine A1M, Ccr levels, and others. In mesangial proliferative glomerulonephritis patients, the expression levels of PAI-1 and TSP-1 in the mesenchyme of glomerular and renal tubules increased with the severity of the disease, suggesting that TSP-1 and PAI-1 are critically involved in the occurrence and development of mesangial proliferative glomerulonephritis.

\section{Acknowledgments}

Funding: None.

\section{Footnote}

Reporting Checklist: The authors have completed the MDAR reporting checklist. Available at http://dx.doi.org/10.21037/ apm-20-1291

Data Sharing Statement: Available at http://dx.doi. org/10.21037/apm-20-1291

Conflicts of Interest: All authors have completed the ICMJE uniform disclosure form (available at http://dx.doi. org/10.21037/apm-20-1291). The authors have no conflicts of interest to declare.

Etbical Statement: The authors are accountable for all aspects of the work in ensuring that questions related to the accuracy or integrity of any part of the work are appropriately investigated and resolved. The study was approved by Ningbo Yinzhou People's Hospital Community (No. 2019-046). All procedures performed in this study involving human participants were in accordance with the
Declaration of Helsinki (as revised in 2013). The informed consent was taken from all the patients.

Open Access Statement: This is an Open Access article distributed in accordance with the Creative Commons Attribution-NonCommercial-NoDerivs 4.0 International License (CC BY-NC-ND 4.0), which permits the noncommercial replication and distribution of the article with the strict proviso that no changes or edits are made and the original work is properly cited (including links to both the formal publication through the relevant DOI and the license). See: https://creativecommons.org/licenses/by-nc-nd/4.0/.

\section{References}

1. Adapa S, Konala VM, Hou J, Naramala S, Agrawal N, Dhingra H, Aronow WS. Seronegative atypical anti-glomerular basement membrane crescentic glomerulonephritis. Ann Transl Med 2019;7:246.

2. Han X, Zheng X, Wang Y, et al. Random forest can accurately predict the development of end-stage renal disease in immunoglobulin a nephropathy patients. Ann Transl Med 2019;7:234.

3. Wang J, Meng X, Li H, et al. Curative effect of valsartan combined with alprostadil in treatment of chronic glomerulonephritis and influence on renal function, hemorheology, t-PA and PAI-1. Hainan Medical Journal 2017;28:4036-9.

4. Pei H, Tong $\mathrm{X}, \mathrm{Yu} \mathrm{H}$, et al. The effect of estimated glomerular filtration rate on outcome of patients with acute ischemic stroke after intravenous thrombolysis with recombinant tissue plasminogen activator. Chinese Journal of Neurology 2018;51:268.

5. Sun J, Yang GH, Liu JX, et al. Discordance Between VASP Phosphorylation and Platelet Aggregation in Defining High On-Clopidogrel Platelet Reactivity After ST-Segment Elevation Myocardial Infarction. Clin Appl Thromb Hemost 2018;24:47-54.

6. Wang H, Pan L, Guo X, et al. Inhibitory Effect of Androgen and Its Receptor on the Expression of Interferon-stimulated Gene 15 in Mouse Spermatocytes. Journal of Medical Molecular Biology 2018;15:13-6.

7. Wang Y, Dong L, Fan Q, et al. The Serum Levels of Soluble Urokinase-type Plasminogen Activator Receptorin Adults with Nephrotic Syndrome. Chinese Journal of Integrated Traditional and Western Nephrology 2017;17:687-91.

8. Yao Y, Chen X, Li J, et al. The Effect of Nursing 
Intervention After Discharged on the Treatment of Nephrotic Syndrome. Journal of Occupational Health and Damage 2017;32:166-8.

9. Tang G, Zheng N, Zhang H, et al. Effects of short-term intensive rosuvastatin on cardiac function, myocardial injury and serum inflammatory factors in patients with acute coronary syndrome after percutaneous coronary intervention. Clinical Journal of Medical Officer 2017;45:89-91.

10. Hu J, Hu L, Yang S. Clinical significance of serum uric acid levels in patients with chronic glomerulonephritis in assessing early and intermediate renal impairment. Modern Practical Medicine 2017;29:1555-7.

11. Xu Y, Xu X, Fang Z, et al. Value of ratio of white blood cell to mean platelet volume and neutrophil to lymphocyte on predicting outcome in acute coronary syndrome with primary percutaneous coronary intervention. Anhui Medical Journal 2017;38:33-7.

12. Yu L, Zhang K, Wang J, et al. The clinical application study of platelet to lymphocyte ratio in type 2 diabetesinduced renal impairment in elderly patients. Chinese Journal of Geriatrics 2018;37:883.

13. Wang P, Qiao H, Wang R. Effect of Hs-CRP Combined with MPV in the Diagnosis of Non-ST-elevation Myocardial Infarction. Journal of Medical Sciencein Central Sauh China 2018;45:597-9.

14. Hu X, Peng H, Liu C, et al. Surveillance Results of Leptospirosis in Dazhou City of Sichuan Province From 2013 to 2015. Journal of Preventive Medicine Information 2017;33:141-3.

15. Wang D. Effect of maixuekang capsule combined

Cite this article as: Pan C, Zhou C, Shan A, Chen N. Expression and clinical significance of thrombospondin-1 and plasminogen activator inhibitor-1 in patients with mesangial proliferative glomerulonephritis. Ann Palliat Med 2020;9(5):31873193. doi: 10.21037/apm-20-1291 with percutaneous coronary intervention on platelet aggregation rate and high sensitivity C-reactive protein in patients with acute coronarysyndrome. Journal of Clinical Medicine in Practice 2018;22:156-7.

16. Tian $\mathrm{R}, \mathrm{Wu} Z$, Wang $\mathrm{H}$, et al. Expression and significance of neutrophil to lymphocyte ratio and platelet to lymphocyte ratio in patients with chronic obstructive pulmonary disease. Clinical Medicine of China 2017;33:433.

17. Cao C, Zhang H, Yang R, et al. Effect of Huoxue Huazhuo capsules on C-reactive protein, platelet aggregation rate and symptom scores in patients with unstable angina. Applied Journal of General Practice 2017;15:1567-9.

18. Koensgen D, Stope MB, Tuerbachova I, et al. Expression, Intracellular Localization, and Prognostic Value of Plasminogen Activator Inhibitor 1 and PAI-1 RNABinding Protein 1 in Primary and Recurrent Ovarian Cancer: A Study of the Tumor Bank Ovarian Cancer Network. Gynecol Obstet Invest 2018;83:508-14.

19. Wang J, Cheng S, Yang X, et al. The effect of Naoxintong capsule treatment on carotid artery intima-media thickness, serum beta thromboglobulin, P-selectin and plasminogen activator inhibitor-I in elderly type 2 diabetic patients with subclinical atherosclerotic vascular disease. Chinese Journal of Geriatrics 2017;36:1080.

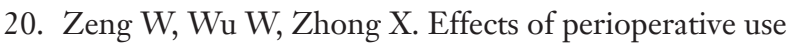
of rhEPO on platelet parameters, hemoglobin levels and rehabilitation progress in patients with hip arthroplasty. Practical Journal of Clinical Medicine 2018;15:87-9.

(English Language Editor: J. Gray) 